\title{
RESONANCE EFFECTS OF ELECTROSTATICALLY ACTUATED ACOUSTIC JETS
}

\author{
Michael O. Muller*, Luis P. Bernal ${ }^{\dagger}$, Peter D. Washabaugh ${ }^{\dagger}$, Hanseup S. Kim', Khalil Najafi ${ }^{\S}$ \\ University of Michigan \\ Ann Arbor, Michigan 48109-2140
}

\begin{abstract}
Acoustic and system resonances of electrostatically driven acoustic jets are studied. At resonance, the jet performance is greatly enhanced, with potential increases of an order of magnitude. An experimental study of a variable geometry acoustic jet is presented: the effects of both the throat and the cavity geometry are studied. Three first mode resonances are found: That of the acoustic cavity; the membrane; and that of the coupled system. The coupled system exhibits a surprisingly different resonance than the individual components. The magnitude of the difference is found to be a function of Reynolds number; the difference is minimized at high Reynolds number.
\end{abstract}

\section{NOMENCLATURE}

$\rho: \quad$ Ambient density

v: Ambient kinematic viscosity

$\rho_{M}: \quad$ Membrane density.

a: $\quad$ Ambient speed of sound.

$\omega_{0}$, fo: Resonant frequency, $(\mathrm{rad} / \mathrm{s}, \mathrm{Hz})$.

$\tau$ : $\quad$ Membrane tension $(\mathrm{N} / \mathrm{m})$.

$r$ : $\quad$ Membrane radial coordinate.

$V_{M}: \quad$ Volume displaced by membrane

$A_{M}: \quad$ Membrane area.

$t_{M}: \quad$ Membrane thickness.

$h_{0}$ : Membrane displacement at center, peak to peak.

$\mathrm{D}_{\mathrm{T}}: \quad$ jet throat diameter.

$A_{T}: \quad$ Geometric jet throat area.

$A_{E}$ : Effective jet throat area,

* Graduate Research Assistant, Department of Aerospace Engineering, Member AIM .

+ Associate Professor, Department of Aerospace Engineering, Senior Member AIAA.

* Graduate Student Research Assistant, Department of Electrical Engineering and Computer Science

$\S$ Professor, Department of Electrical Engineering and Computer Science.

Copyright (C) 2003 by Michael O. Muller, Published by the American Institute of Aeronautics and Astronautics, Inc. with permission accounting for viscous blockage;

$\mathrm{L}_{\mathrm{T}}: \quad$ Geometric jet throat length.

$\mathrm{L}_{\mathrm{E}}$ : Effective jet throat length, accounting for the inertia of the fluid in motion inside the throat,

$V_{c}$ : Acoustic cavity volume.

$h_{\mathrm{C}}$ : Acoustic cavity height.

$\mathrm{D}_{\mathrm{C}}$ : Acoustic cavity diameter.

$\mathrm{u}_{\mathrm{E}}$ : Peak jet throat velocity.

\section{INTRODUCTION}

This paper describes the experimental characterization of the resonant behavior of an electrostatically actuated acoustic jet at varying scales. Resonance occurs in individual system elements as well as in the coupled system. Resonance of the acoustic cavity manifests itself as a maximum in the peak jet throat velocity, corresponding to a maximum jet impulse. Resonance of the electrostatically actuated membrane corresponds to the frequency of maximum oscillation amplitude for a given input excitation voltage. Resonance of the coupled system is determined in the same manner as that of the acoustic cavity, namely by the maximum jet thrust performance for a given electric input to the driving electrode.

The use of electrostatic actuation is motivated by micro scale applications, employing MEMS fabrication technologies.' Acoustic jets can provide a propulsion technology for very small scale systems. ${ }^{2}$ At micro-scales, electrostatic actuation of the acoustic jet is more effective compared to other actuation technologies because of the increase in surface area to volume ratio with reduced size. The insights gained in the present investigation are relevant to an ongoing effort in the design of high impulse acoustic jets for flow control and propulsion applications.

Analytic theory of acoustic jets have been reported by Muller et $a l^{2,3}$ and more recently by Gallas et al. ${ }^{4}$ Theory shows that maximum performance is obtained when the acoustic cavity is operated at resonance. Here the velocity calculated based upon just the volume displaced

1

American Institute of Aeronautics and Astronautics 
by the membrane, where the fluid is assumed to be incompressible, is called "incompressible." The throat velocity of an acoustic jet operating at resonance can be well above the calculated "incompressible" velocity.

Analysis of the entire system ${ }^{3}$ including the electrostatically driven membrane and acoustic cavity shows that maximum performance of the coupled system occurs at a frequency different from both the acoustic cavity resonant frequency and the structural resonant frequency of the membrane. A non-dimensional coupling parameter, $K_{D}$, defined as the ratio of the acoustic pressure load acting on the membrane to an equivalent pressure due to the membrane inertia, given by equation 1 , determines the coupling dynamics. For small $K_{D} \ll 1$ the acoustic cavity resonance and mechanical resonance of the membrane are effectively uncoupled. For $K_{D} \geq 1$ there is strong coupling between the cavity acoustic field and the membrane motion.

$K_{D}=\frac{\rho}{\rho_{M} t_{M}}\left(\frac{a}{\omega_{0}}\right)^{2} \frac{2 A_{M}}{V_{M}}$

The present investigation was initiated to substantiate these theoretical predictions and investigate the nature of viscous effects and associated performance loses. Tests are conducted at scales sufficiently large to facilitate instrumentation and measurement resolution while maintaining the range of non-dimensional parameters relevant to micro-scale implementation. An axisymmetric jet configuration is used to eliminate the secondary effects of more complex geometries typical of MEMS devices.

The acoustic cavity and membrane resonances are first investigated separately, followed by an investigation of coupling effects of the complete system. The effects of varying geometry are explored where applicable: the cavity geometry does not affect the isolated membrane. By varying the jet throat diameter, both the jet Reynolds number and the resonant frequency are varied. By varying the resonator volume, the cavity resonant frequency is varied while maintaining the throat Reynolds number. In this paper the throat Reynolds number is defined by equation 2 , which is sometimes called the Stokes number in the literature. $^{4}$

$\operatorname{Re}_{f_{-}}=\underline{f_{0} D} D_{T}^{2}$

\section{EXPERIMENTAL |}

Schematics of the apparatus, showing the design details, are shown in Figures 1 and 2. It consists of four main components which, using the same labels as in the figures, are: (a) the throat plate, (b) the cavity spacer, (c) the electrode assembly and (d) the membrane assembly. The throat plate is a removable disk that is used to vary the throat diameter, $D_{T}$. The cavity spacer is a removable cylinder that is used to vary the cavity height, $h$ and thereby the cavity volume, $V$, The electrode assembly is a mandrel against which the membrane is tensioned. It also houses the perforated electrode (in this case a stainless steel honeycomb with $1 / 16$ cells) and a fiberoptic noncontacting and non-conducting position sensor. The membrane assembly consists of an aluminum membrane sandwiched between two 1/8" aluminum

rings.

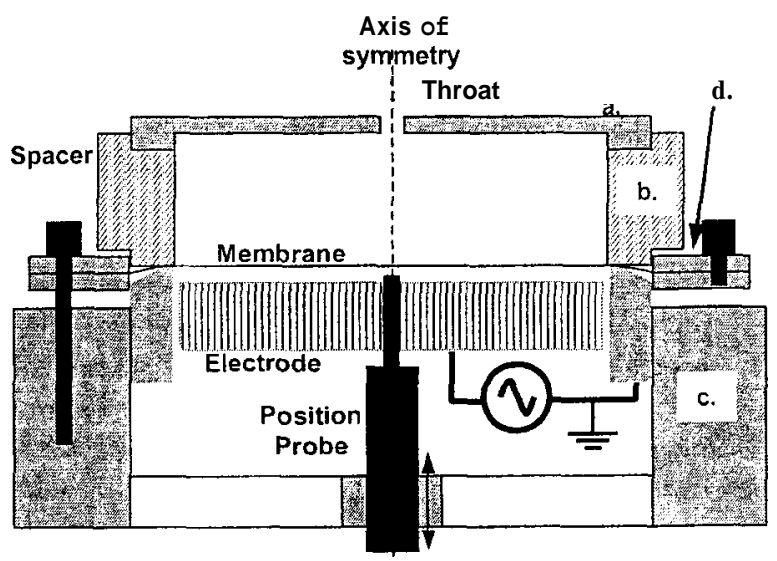

FIGURE 1: Cross sectional view of the acoustic jet apparatus. The axis of symmetry is vertical and centered in the throat, as indicated. The membrane tension is adjusted with long bolts on the periphery; the alternating short bolts clamp the membrane in position. The position probe can be moved vertically, allowing calibration in situ. The letters correspond to those shown in figure 3.

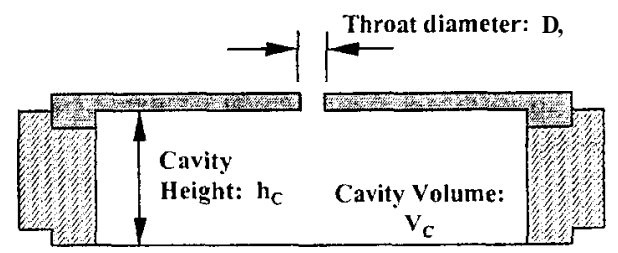

FIGURE 2: Schematic of the cavity, indicating the geometric nomenclature.

This modular construction allows exploration of a range of performance parameters with relative ease. The dimensions are such that the Reynolds 
number at resonance is matched to MEMS devices under development, and is of order $10^{2}$. Figures 3 and 4 show photographs of the components and a typical assembly, respectively.

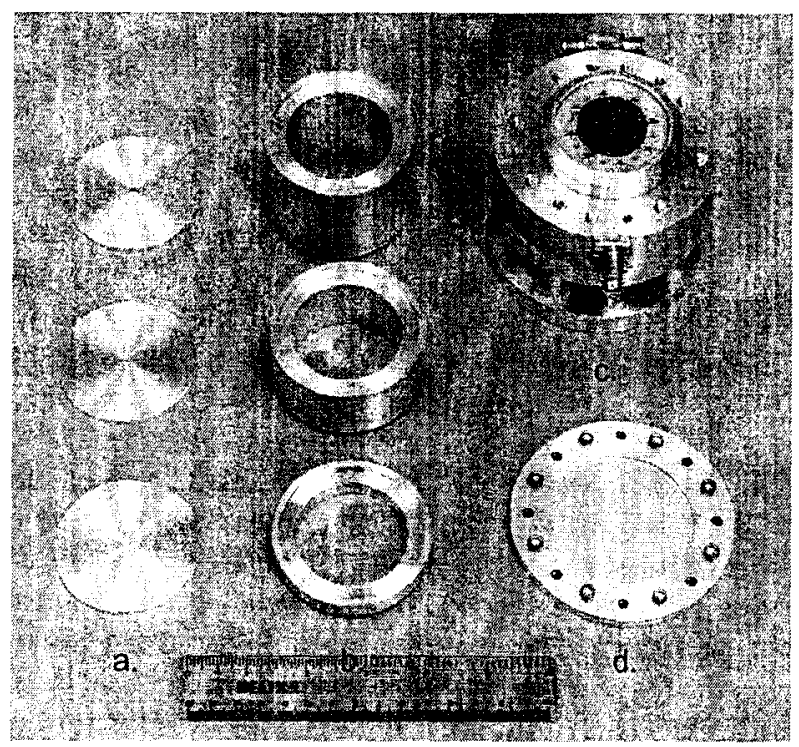

FIGURE 3: Photograph of the jet components. On the left (a) are three different throat plates. In the center (b) are three different cavity spacers, used to define the cavity volume. On the right (c) is the electrode assembly, containing the honeycomb electrode at its center. The BNC connector visible at the front of the base connects to the electrode. Also shown, below the base (d), is the membrane assembly.

The membrane is a $16.5 \mu \mathrm{m}$ thick aluminum foil. A fiberoptic position probe located at the center of the electrode measures the membrane deflection. The position probe is calibrated in situ for every test, as the reflectivity of the foil was found to be variable. It should be noted that the position probe measurements are only valid for the fundamental membrane mode. For higher order modes, the membrane surface at the center is not necessarily normal to the probe, in which case the indicated displacement is larger than the true value. For the present tests, multiple membranes were used. The tension between individual membranes is controlled via fixed assembly methods, and is confirmed using a static (DC) deflection test. Although possible, adjustment of the membrane tension was not required after installation in the apparatus.

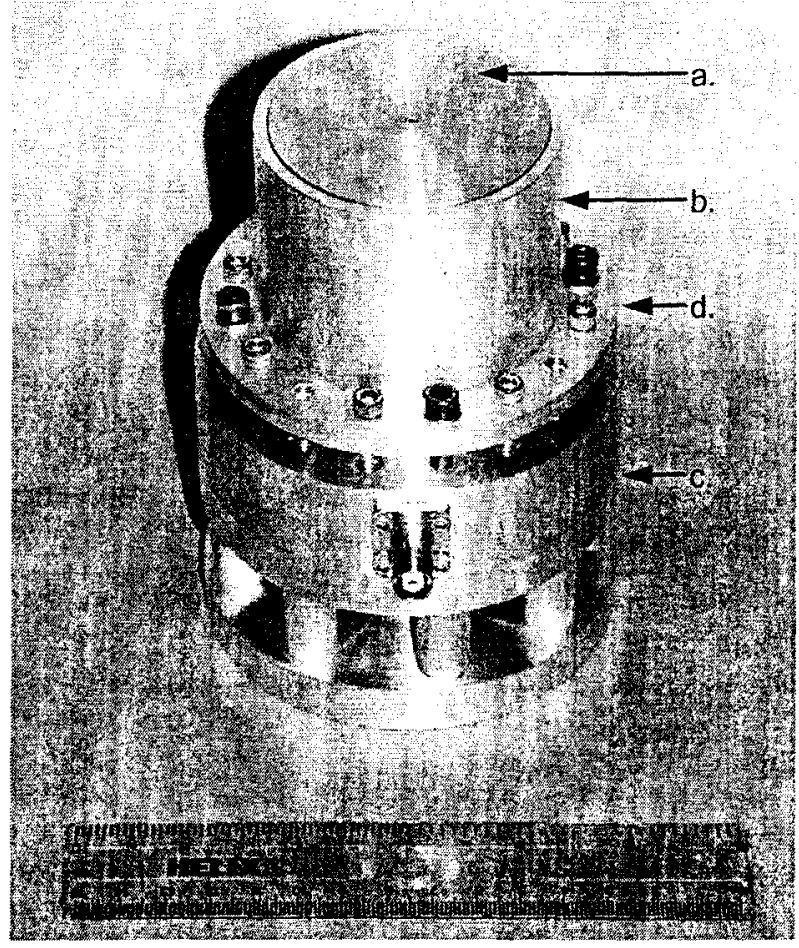

FIgURE 4: Photograph of assembled apparatus.

Actuation of the membrane is by a sinusoidal $A C$ voltage of up to $800 \mathrm{~V}_{\text {o-p }}$. The membrane itself is grounded; the drive voltage is applied to a parallel steel honeycomb electrode. There is no insulation between the membrane and electrode, and at higher voltages the electrode arcs to the membrane resulting in zero applied force and thus no appreciable deflection. As with electrostatic actuation common in MEMS devices, the actuation frequency is double the frequency of the drive voltage. This frequency doubting is because the electrostatic pressure is proportional to the electric field squared.

As described above the cavity volume is defined by the length of the cavity spacer element on top of the membrane. For the present series of tests, the cavity volume is adjusted in multiples of four. The resonant frequency is a function of the inverse square of the cavity volume ${ }^{2}$ :

$f_{0}=\frac{a}{-2 \pi} \sqrt{\frac{A_{E}-}{L_{E} V_{C}}}$

Thus, using three different cavity volumes with the maximum volume being 16 times the minimum volume, the resonant frequency is varied by a factor of four.

Varying the cavity volume changes the resonant frequency of the jet without affecting the 
throat Reynolds number. However, by using a variable throat diameter, the jet Reynolds number can be changed (simultaneously changing the acoustic resonant frequency). Three different throat diameters are used, each larger than the previous by a factor of two, such that the cavity resonant frequencies is varied by a factor of four.

The throat velocity is measured with a hot wire, placed above the throat, at a downstream distance less than 0.1 throat diameters. The minimum velocity measurable by the hot wire is approximately $0.15 \mathrm{~m} / \mathrm{s}$, limited by the resolution of the data acquisition system.

Assembly of the apparatus is accomplished by first bolting the membrane tensioning ring to the base. The membrane tension is controlled by tightening the tensioning ring; again, by consistent assembly protocol the membrane tension is repeatable. The cavity and throat are laid upon the membrane, using silicon vacuum grease to seal the cavity. Gravity, plus the stick of the silicon grease is sufficient to maintain the cavity integrity at the pressures produced by the membrane motion.

In summary, three different volumes and throats were tested, for a total of nine cases, as shown in Table 1. This table lists the parameters $A_{E}$ and $L_{E}$ used to calculate the resonant frequency of the cavity given by equation 2 .

\begin{tabular}{|c|c|c|c|c|c|}
\hline Case & $\begin{array}{c}h_{c} \\
10^{-3} \mathrm{~m}\end{array}$ & $\begin{array}{c}\mathrm{DT}_{\mathrm{T}} \\
(m x \\
\left.10^{-3}\right)\end{array}$ & $\begin{array}{c}V_{c} \\
\left(m^{3} x\right. \\
\left.10^{-6}\right)\end{array}$ & $\begin{array}{c}A_{E} \\
\left(m^{2} x\right. \\
\left.10^{-6}\right)\end{array}$ & $\begin{array}{c}L_{E} \\
(m x \\
\left.10^{-3}\right)\end{array}$ \\
\hline 1 & 2.54 & 0.58 & 4.65 & 0.268 & 1.09 \\
\hline 2 & & 1.22 & & 1.17 & 1.59 \\
\hline 3 & & 2.46 & & 4.77 & 2.57 \\
\hline 4 & 12.7 & 0.58 & 23.2 & 0.268 & 1.09 \\
\hline 5 & & 1.22 & & 1.17 & 1.59 \\
\hline 6 & & 2.46 & & 4.77 & 2.57 \\
\hline 7 & 53.3 & 0.58 & 864 & 0.268 & 1.09 \\
\hline 8 & & 1.22 & & 1.17 & 1.59 \\
\hline 9 & & 2.46 & & 4.77 & 2.57 \\
\hline
\end{tabular}

TABLE 1: Acoustic jet geometry. For all cases the cavity diameter (equal to the membrane diameter) $D_{C}=48.3 \times 10^{-3} \mathrm{~m}$, the throat length $L_{T}=6.35 \times 10^{-4}$ $m$, and the membrane mass per unit area, $\rho_{M} \times t_{M}$, is $4.462 \times 10^{-2} \mathrm{~kg} / \mathrm{m}^{2}$.

\section{RESULTS}

\section{ACOUSTIC CAVITY}

Measurements of the throat velocity at constant membrane deflection amplitude and varying frequency, determine the resonance characteristics of the acoustic cavity. The constant actuation amplitude effectively removes the membrane dynamics from the performance of the resonator. The membrane actuation is $1.5 \pm$ $0.1 \mu \mathrm{m}$, peak to peak. This choice of deflection amplitude is dictated by the maximum possible deflection across the full frequency range, up to $1,000 \mathrm{~Hz}$. The limit on deflection is the previously mentioned maximum possible voltage, namely the breakdown voltage of the electrode to membrane gap. The error band of $0.1 \mu \mathrm{m}$ is determined by the noise level of the position probe, as well as the accuracy required to observe the resonant phenomena. This value is small enough to clearly show the resonant behavior. Plots of the throat velocity as a function of frequency for cases 4,5 and 6 in Table 1 are shown in figure 5.

Also shown in the figure is the calculated incompressible orifice velocity, based on the measured motion of the center of the diaphragm. It is assumed that the membrane motion is given by its first mode, described by a Bessel function of position and harmonic function in time: ${ }^{5}$

$$
y(r, t)=h_{0} J_{0}\left(k_{0,1} 2 r / D_{M}\right) \cos \left(\omega_{0,1} t\right)
$$

where,

$$
\begin{aligned}
& \mathrm{k}_{0,1}: \quad \begin{array}{l}
\text { First zero of the } 0^{\text {th }} \text { order Bessel } \\
\text { function }(=2.405)
\end{array} \\
& \omega_{0,1}: \begin{array}{l}
\text { Resonant frequency of the } \\
\text { fundamental mode. }
\end{array}
\end{aligned}
$$

and the subscripts refer to the azimuthal and radial mode index, respectively. Given this volume displacement, and the period the peak throat velocity is calculated. It is important to note that this assumed mode is only relevant for the first natural frequency. Higher order modes have different shapes.

This calculated incompressible velocity is almost straight diagonal line plotted in the figure. At resonance, the actual velocity can significantly exceed the calculated incompressible velocity. 


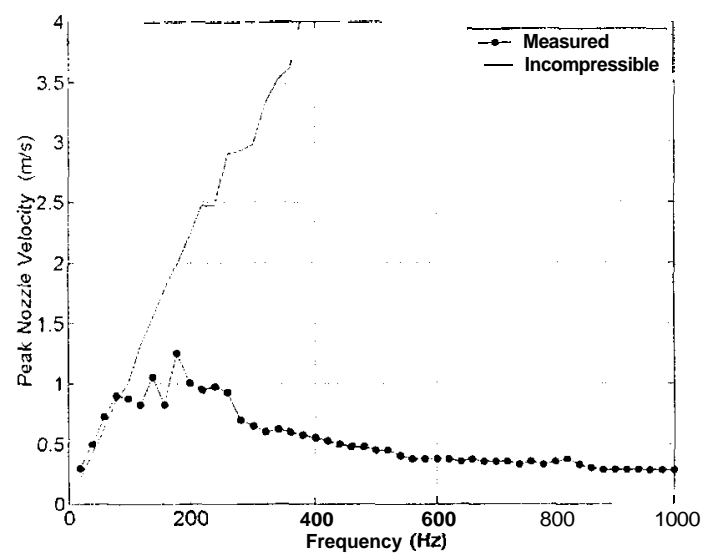

(a)

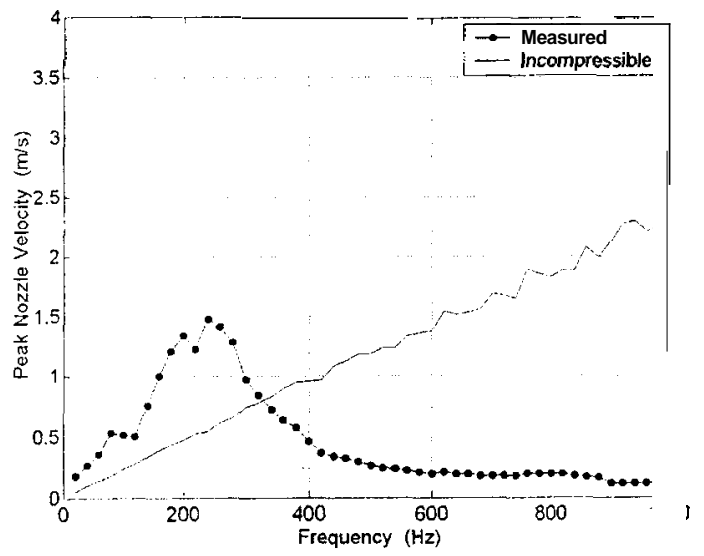

(b)
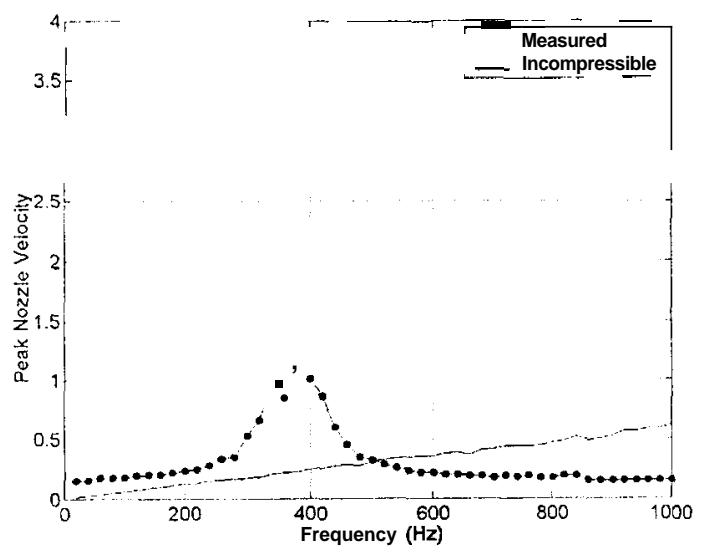

(c)

FIGURE 5: Peak throat velocity at constant membrane displacement. "Measured" curves show the actual peak throat velocity. "Incompressible" curves are the calculated velocity based on measured membrane motion. Departure from a straight line of the "incompressible"curves indicates the variability in the membrane displacement. Cases 4, 5 and 6 shown.
Table 2 lists the resonant frequency of the individual components of the system at the test conditions listed in Table 1.

\begin{tabular}{|c|c|c|c|c|c|}
\hline & $\mathrm{f}_{\mathrm{o}}$ & $\mathrm{U}_{\text {e. } \max }$ & $\begin{array}{c}\mathrm{U}_{\text {e. Inc. }} \\
\max \end{array}$ & $\mathrm{C}_{\mathrm{u}}$ & $\mathrm{Re}_{\mathrm{fo}}$ \\
Case & $\mathbf{H z}$ & $\mathrm{m} / \mathrm{s}$ & $\mathrm{m} / \mathrm{s}$ & $\%$ & \\
\hline 1 & 220 & 1.828 & 2.324 & 79 & 5.01 \\
2 & 300 & 1.837 & 0.7162 & 260 & 29.7 \\
3 & 540 & 0.9081 & 0.3171 & 290 & 219 \\
\hline 4 & 180 & 1.257 & 1.901 & 66 & 4.10 \\
5 & 240 & 1.475 & 0.5730 & 260 & 23.8 \\
6 & 380 & 1.099 & 0.2231 & 490 & 154 \\
\hline 7 & 80 & 0.7539 & 0.8540 & 88 & 1.82 \\
8 & 140 & 1.187 & 0.3343 & 360 & 13.9 \\
9 & 220 & 1.069 & 0.1292 & 830 & 89.0 \\
\hline
\end{tabular}

TABLE 2: Uncoupled acoustic cavity resonance and throat velocity at resonancefor all nine cases listed in table 1 . Shown are the measured peak throat velocity, the calculated incompressiblepeak throat velocity based on membrane deflection, and the calculated velocity overshoot and Reynolds number Predicted resonantfrequency and peak throat velocity are also shown. The system is driven at constant membrane amplitude.

\section{MEMBRANeRESONANCE}

The membrane structural dynamics is determined by measurements of the spectrum of the membrane displacement in vacuum. This test isolates the membrane dynamics from any damping of the surrounding air and/or acoustic cavity effects. Because structural damping is very low, the resulting dynamics is a pure mass-spring system. From this test, the membrane first resonant frequency is determined. Given the membrane density and thickness, and assuming a first mode of vibration, the tension can be calculated $^{5}$ :

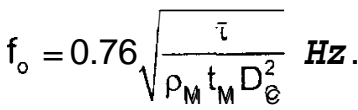

For the present test the membrane tension is held constant. Figure 6 shows the membrane spectrum in vacuum. Evident is a peak at 1140 $\mathrm{Hz}$. Given a membrane mass per unit area of 4.46 $\times 10^{-2} \mathrm{~kg} / \mathrm{m}^{2}$, the resulting tension is $234 \mathrm{~N} / \mathrm{m}$. 
There is also a peak at very low frequency that is associated with the DC voltage bias of fiberoptic position sensor and does not represent motion of the membrane.

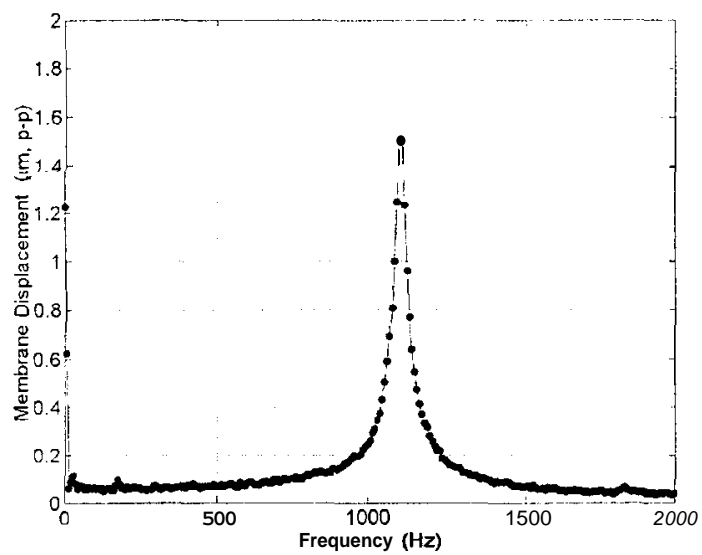

FIGURE 6: Membrane position response in vacuum. $f_{0}=1140 \mathrm{~Hz}$. Coupled System

\section{COUPLED SYSTEM}

The final element of the experimental investigation is the complete acoustic jet system, namely the acoustic cavity driven by the electrostatically actuated membrane. To investigate the coupling effects, the membrane is driven at constant voltage and varying frequency. The same conditions given in Table 1 were used in these tests with a constant membrane tension.

Spectra of the coupled membrane and acoustic cavity system are shown in Figure 7 . The data shown are for cases 4, 5 and 6. Each plot in the figure shows the peak throat velocity, overlaid with the calculated incompressible throat velocity, determined from the position probe measurements. The throat velocity data show a resonance at lower frequencies than observed in the spectra of the isolated acoustic cavity.

The membrane position data also show peaks at higher frequencies. It is important to note that due to the measurement characteristics of the fiberoptic probe, as discussed above in the "Experimental Apparatus" section, the high frequency peaks in these measurements may be due to non-axisymmetric motion of the membrane or higher order modes. For frequencies below the structural resonant frequency measured in vacuum, the first vibrational mode of the membrane is expected.

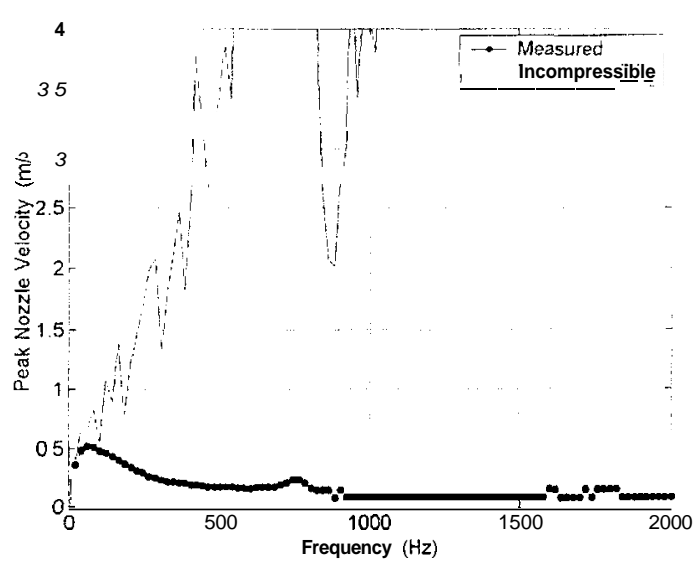

(a)

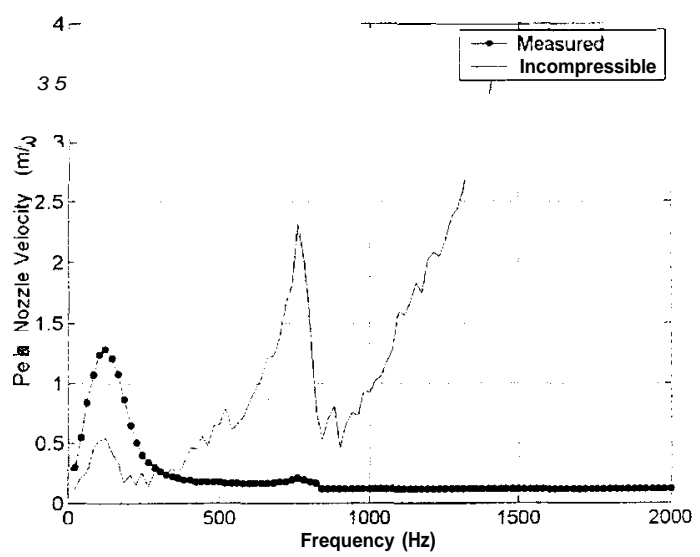

(b)

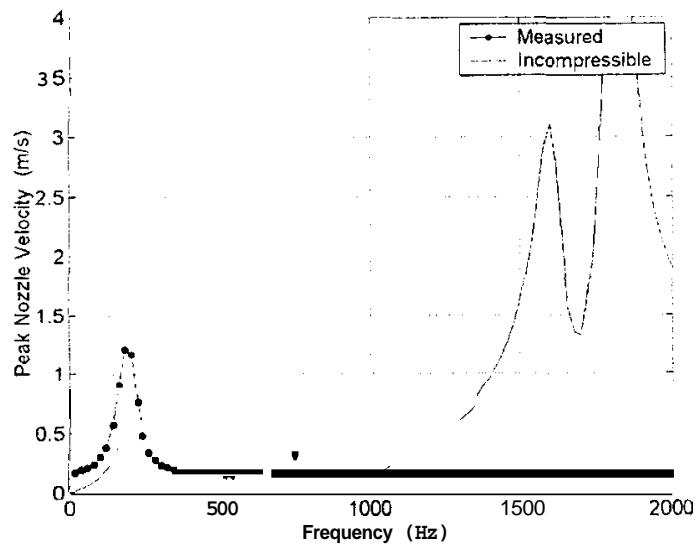

(c)

FIGURE 7: Peak throat velocity corresponding to membrane deflection of figure 5 . Cases 4,5 and 6 . The minimum threshold for the hotwire is $0.15 \mathrm{~m} / \mathrm{s}$. Above $-850 \mathrm{~Hz}$, velocity is effectively zero.

At higher frequency, higher order modes are expected which may or may not have nodes at the center, but since the position sensor only measures the center displacement, the membrane 
motion cannot be determined uniquely from the single probe output. For example while higher order modes can have a displacement at the center, they will have opposite displacement at other positions. Thus the assumption of a first mode deformation (as is done in the incompressible calculation) will result in velocities much larger than those actually present. This explains the large discrepancy between the calculated and measured velocities at frequencies larger than the first resonance.

\begin{tabular}{|c|c|c|c|c|c|}
\hline Case & $\begin{array}{c}f_{0} \\
\text { Membrane } \\
H z\end{array}$ & $\begin{array}{c}f_{c p} \\
\text { Cavity } \\
H z\end{array}$ & $\begin{array}{c}\mathrm{u}_{\mathrm{e}, \max } \\
\mathrm{m} / \mathrm{s}\end{array}$ & $\begin{array}{c}\mathrm{U}_{\mathrm{e}, \mathrm{Inc}} \\
\max \\
\mathrm{m} / \mathrm{s}\end{array}$ & $\begin{array}{l}\mathrm{C}_{\mathrm{u}} \\
\%\end{array}$ \\
\hline 1 & $<20$ & 80 & 0.9887 & 0.7786 & 130 \\
\hline 2 & 80 & 120 & 1.350 & 0.6720 & 200 \\
\hline 3 & 280 & 300 & 0.9797 & 0.5377 & 180 \\
\hline 4 & $<20,760$ & 80 & 0.5136 & 0.8450 & 61 \\
\hline 5 & 80,760 & 120 & 1.2835 & 0.5406 & 240 \\
\hline 6 & 180,780 & 180 & 1.2164 & 0.5266 & 230 \\
\hline 7 & 640 & 60 & 0.4736 & 0.5078 & 93 \\
\hline 8 & 640 & 100 & 0.8940 & 0.1712 & 520 \\
\hline 9 & 160,660 & 160 & 0.8206 & 0.1343 & 610 \\
\hline
\end{tabular}

TABLE 3: Coupled acoustic cavity resonance and throat velocity at resonance for all nine cases listed in Table 1. The system is driven at a specified voltage amplitude.

The results of the coupled system tests are summarized in Table 3. These data show strong coupling between the membrane and the acoustic cavity resonances. The resonant frequency of the system is different from the individual resonances of each the components. Furthermore, there are multiple peaks in the position probe output spectra $\left(2^{\text {nd }}\right.$ column $)$ in Table 3 that are not present in the isolated components. For the largest throat diameters (cases 3, 6 and 9), the coupled cavity resonance coincides with one of the resonances of the coupled system. For the small throat diameters (cases 1, 4 and 7), the two resonances are significantly different. The membrane deflection is non-zero away from its resonance, and is sufficient to excite the cavity resonance for the smaller throat diameters.

\section{DISCUSSION}

\section{$\underline{\text { ACOUSTIC CAVITY }}$}

Four dimensionless numbers are used to describe the performance of the acoustic cavity. The Reynolds number at resonance is as shown in equation 2. A non-dimensional frequency, $C_{f}$, relates the resonant frequency to the diameter and acoustic speed, as shown in equation 6 . The velocity overshoot ratio, $C_{u}$, is defined as the ratio of measured velocity at resonance to the calculated incompressible velocity based on membrane volume displacement, as shown in equation 7. This parameter describes the compressible nature of the flow at resonance, and is a measure of the performance gain obtained when operating at the cavity resonance instead of off-resonance. The volume ratio, $V_{R}$, of equation 8 is based on dimensional arguments, as discussed below in equations 9 and 10 .

$C_{f}=\frac{f_{0} D_{T}}{a}$

$\mathrm{C}_{\mathrm{u}}=\frac{\mathrm{u}_{e, \text { rnax }}}{\mathrm{u}_{\mathrm{e}, \text { max:incompressible }}}$

$V_{R}=\frac{D_{T} A_{T}}{V_{C}}$

Table 2 summarizes the experimental results for all nine cases considered in this investigation. Shown are the measured cavity resonance frequency, $f_{0}$, the peak throat velocity at resonance, $U_{e}$, max, the calculated incompressible peak throat velocity at resonance, $u_{e}$ inc., max, and the overshoot ratio, $\mathrm{G}$

The results of the present measurements in terms of these parameters are plotted in figures 8 and 9 . The plots show good agreement between expected and measured results. Figure 8 shows the $\mathrm{C}_{\mathrm{u}}$ ratio as a function of the resonant Reynolds number. Evident is a dependence of the velocity overshoot on the Reynolds number. This indicates that to increase the performance of an acoustic resonator, the Reynolds number should be maximized.

The relation between $C_{f}$ and $V_{R}$ can be derived from theory. The resonant frequency of an acoustic cavity given by equation 3 can be written as: 


$$
\begin{aligned}
& C_{f}=\frac{f_{0} D_{T}}{a}=\frac{D_{T}}{2 \pi} \sqrt{\frac{A_{E}}{L_{E} V_{C}}}= \\
& \frac{1}{2 \pi} \sqrt{\frac{D_{T}}{L_{E}}} \sqrt{\frac{D_{T} A_{T}}{V_{C}}}
\end{aligned}
$$

thus,

$$
\mathrm{f}_{\mathrm{O}} \mathrm{D}_{\mathrm{T}} \sqrt{\frac{\mathrm{D}_{T} \mathrm{~A}_{T}}{V_{C}}}
$$

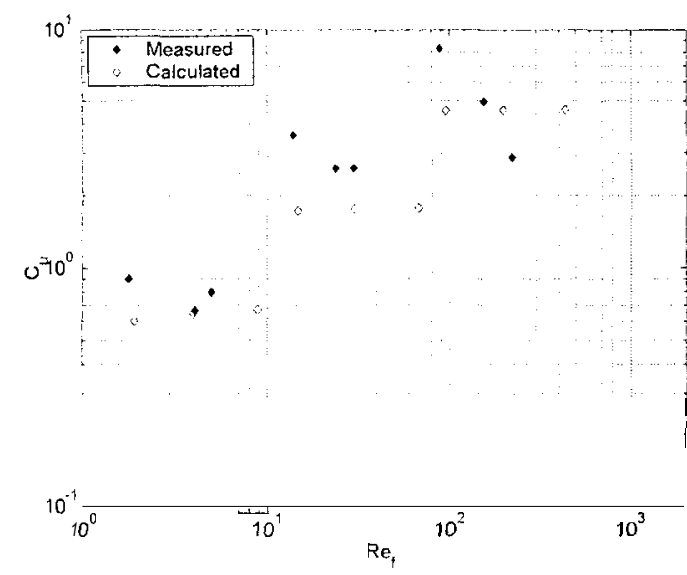

FIGURE 8: Ratio of the actual peak nozzle velocity at resonance to the calculated incompressible velocity based on membrane displacement, as a function of the resonance Reynolds number. Solid and open markers indicate experimental data and analytic predictions, respectively.

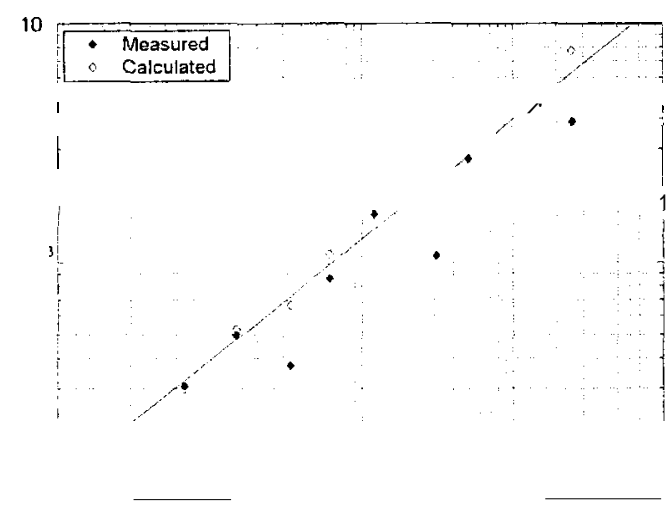

FIGURE 9: Dimensionless parameters $C_{f}=f_{0} D_{T} / a$ as a function of $V_{R}=A_{T} D_{T} N_{C}$. Solid and open markers indicate experimental data and analytic predictions, respectively.

This square root relation in equation 10 is observed in the data, as indicated by the solid line in figure 10. The three points below the general trend correspond to cases 1 through 3 , employing the smallest cavity. For these cases, the ratio of cavity depth to nozzle diameter is about unity. The data is consistent with a smaller value of $D_{T} / L_{E}$ for these cases. This suggests an increase in the effective length of the throat, $L_{E}$, for small values of the cavity depth to throat diameter ratio. The resonant frequency, as given by equation 3 decreases as the square root of the effective length. Corrections of this nature would shift the predicted data closer to the measured values.

\section{Coupled System}

Table 3 shows the values at resonance for the coupled experiments, tabulating the same values as in Table 2. For cases 1 and 4 , the first mechanical resonance, namely a peak in the displacement magnitude, of the membrane is below $20 \mathrm{~Hz}$, and thus not fully resolved in the data. Evident is a first structural frequency an order of magnitude less than the uncoupled resonance.

Examining the acoustic resonances, also the frequency of resonance is much lower than for the uncoupled acoustic cavity. The velocity overshoot ratio, $\mathrm{C}_{w}$ is similar in magnitude, indicating a similar dependence on the Reynolds number.

It is interesting to compare the present experimental results with the analysis of a coupled, electrostatically actuated acoustic jet. ${ }^{3}$ As mentioned earlier the parameter $K_{D}$ (the ratio of the acoustic pressure load acting on the membrane to an equivalent pressure due to the membrane inertia) determines the nature of the coupling between the membrane motion and the acoustic cavity. For the present test $K_{D}$ is of order $10^{6}$, owing to the low cavity resonant frequency and very thin membrane. For this very large value of $\mathrm{K}_{\mathrm{D}}$ the membrane inertia or stiffness cannot balance the acoustic or electrostatic pressure force and therefore the acoustic pressure must equal the electrostatic pressure acting on the membrane at all times. Thus the membrane moves very quickly to the equilibrium position given by the condition that the electrostatic pressure equals the acoustic pressure. This increases the apparent mass of the system. A linear analysis of the coupled system in the limit $K_{D} \gg 1$ gives the resonant frequency as: 


$$
f_{C P}=\frac{f_{0}}{\sqrt{1+\frac{\rho a^{2} A_{M}}{V_{C} \rho_{M} t_{M} \omega_{M}^{2}}}}=\frac{\frac{a}{2 \pi} \sqrt{\frac{A_{c}}{L_{E} V_{C}}}}{\sqrt{1+\frac{\rho a^{2} A_{M}}{V_{C} \rho_{M} t_{M} \omega_{M}^{2}}}}
$$

where $f_{c p}$ is the coupled system resonant frequency in $H z, f_{0}$ is the uncoupled cavity resonant frequency in $\mathrm{Hz}$, and $\omega_{M}$ is the uncoupled membrane resonant frequency in rad/s. Figure 10 is a plot of the nondimensional frequency of the coupled system, $C_{\text {fcp }}=f_{\text {cp }} D_{T} / a$, as a function of the volume coefficient, $V_{R}$. Experimental data are shown as solid symbols and calculated results using equation 11 are the open symbols. There is excellent agreement between theory and experiments.

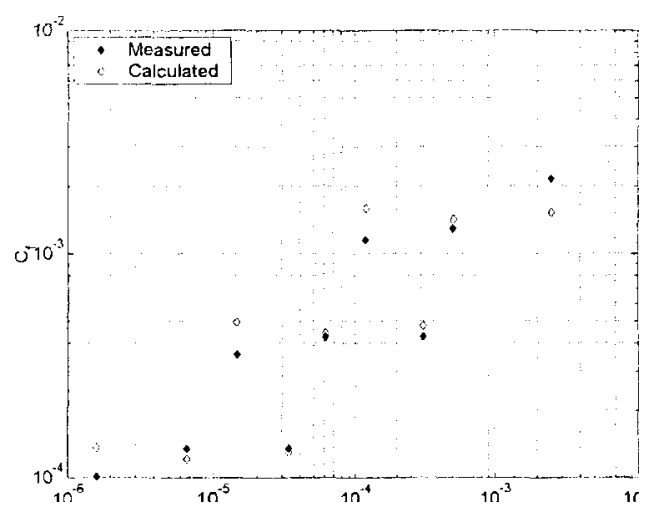

FIGURE 10: Dimensionless parameters $C_{f}=f_{C P} D_{T} /$ a as a function of $V_{R}=A_{T} D_{T} N_{C}$. Solid and open markers indicate experimental data and analytic predictions, respectively.

in summary these results show that the membrane dynamics still play a role in the resonant frequency of the coupled system. Increasing the structural resonant frequency increases the coupled system resonant frequency. Also the velocity overshoot ratio is lower in the coupled system case primarily due to the lower resonant frequency. It should be noted that $K_{D}$ for current MEMS designs is significantly lower than in the present tests because of the much larger cavity frequency.

\section{CONCLUSION}

In this paper, the resonance effects of electrostatically actuated acoustic jets are experimentally documented. The performance of the acoustic jet compares favorably to analytic analysis. The behavior of the electrostatic drive, and how it relates to the acoustic resonator performance, is discussed. Resonance is extremely beneficial to the performance of acoustic jets. A velocity gain of almost tenfold is seen above the calculated incompressible velocity produced by the membrane deflection. From a design perspective, this performance gain increases with increasing Reynolds numbers. There is some evidence of the benefits of a deep cavity. Cavity depth an order of magnitude larger than throat diameter results in higher operating frequency and exit velocity.

The electrostatically actuated drive membrane is also highly resonant, with a very high quality factor. The coupled system produces resonances significantly different than the individual component resonances. This is consistent with previous models. ${ }^{3}$

\section{ACKNOWLEDGEMENTS}

Research sponsored by DARPA Contract N00019-98-KO111, and the Engineering Research Centers Shared Facilities supported by the National Science Foundation under Award Number EEC-0096866.

\section{REFERENCES}

${ }^{1}$ Chou, T.-K.A., Najafi, K., MUller,M.O., Bernal, L.P. AND WASHABAUGH,P.D. 2001 High Density Micromachined Acoustic Ejector Arrays for Micro Propulsion. Transducers 2007.

${ }^{2}$ Müller, M.O., Bernal, L.P., Moran, R.P., Washabaugh,P.D.,Parviz, B.A. And Najafi,K. 2000 MicromachinedAcoustic Resonators for Microjet Propulsion. AIAA Paper 2000-0547.

' Müller, M.O., Bernal, L.P., Moran, R.P., Washabaugh,P.D., PaRVIZ,B.A., ChOU.T.-K.A., Zhang, C. AND Najafi, K. 2000 Thrust Performance of Micromachined Synthetic Jets. AIAA Paper 2000-2404.

${ }^{4}$ Gallas, Q., Matheew,J., Kaysap, A., Holamn,R., NISHIOA,T., CARROЦB., SHEPLAK, M. AND CATTAFESTA, L. 2002 Lumped element modeling of Piezoelectric-DrivenSynthetic Jet Actuators. AIAA Paper 2002-0125.

5 MEIROVITCH,L. 1967 Analytical Methods in Vibrations. MacmillanPublishing Co., Inc. 
